\title{
Validación de un algoritmo para inferir función ventricular izquierda preservada luego de un infarto de miocardio.
}

\author{
Tobin K., Stomel R., Harber D. y col. Arch. Intern. Med. 1999, 159: 353-357
}

\begin{abstract}
Objetivo
Validar un algoritmo compuesto de 4 criterios (clínicos y/o electrocardiográficos) para la predicción de una fracción de eyección de al menos $40 \%$ en pacientes post-infarto agudo de miocardio.
\end{abstract}

\section{Diseño}

Estudio de cohorte retrospectivo.

Lugar

Hospital de Comunidad (330 camas) de Michigan, USA.

\section{Pacientes}

Pacientes consecutivos admitidos con diagnóstico de infarto aqudo de miocardio que tuvieran una medición de la fracción de eyección, ingresados entre junio de 1993 a marzo de 1995. De un registro inicial de 293 pacientes, fueron excluidos: 65 sin criterios diagnósticos de infarto, 14 sin medición de la fracción de eyección, y 1 por imposibilidad de revisión del estudio de medición.

\begin{abstract}
Descripción de los tests y del test de referencia
La fracción de eyección del ventrículo izquierdo fue evaluada con una o más de las siguientes técnicas: ecocardiograma transtorácico, ventriculograma con contraste, ó ventriculograma radioisotópico. Todas las mediciones fueron revisadas por dos cardiólogos sin conocimiento de los datos clínicos del paciente. El algoritmo de predicción de función ventricular preservada se basó en 1 variable clínica (presencia o ausencia de insuficiencia cardíaca preexistente o durante el infarto en curso) y en 3 variables electrocardiográficas: 1) electrocardiograma interpretable (ausencia de bloqueo de rama izquierda, ritmo de marcapasos, o hipertrofia ventricular izquierda con patente de sobrecarga), 2) ausencia de ondas $Q$ en una localización electrocardiográfica diferente a la correspondiente al infarto actual y 3) ausencia de ondas $Q$ de localización anterior en el infarto actual.
\end{abstract}

\section{Medición de resultados}

Determinación de la exactitud diagnóstica (valor predictivo positivo y error predictivo) del algoritmo evaluado para la predicción de una fracción de eyección $\geq 40 \%$ en pacientes post-infarto agudo de miocardio.

\section{Resultados principales}

De los 239 pacientes con infarto de miocardio evaluados, 127 eran hombres y 86 mujeres, siendo la edad media de $67+/-13$ años. En 90 pacientes el infarto era de tipo Q $(42,3 \%)$, habiendo recibido tratamiento trombolítico el 71\%. La evaluación de la fracción de eyección se efectúo en 167 pacientes por ecocardiograma, en 48 por ventriculograma con contraste y en 5 por ventriculograma radioisotópico. En 83 pacientes el algoritmo pudo ser utilizado para predecir una función ventricular conservada. Setenta y uno de los mismos presentaron una fracción de eyección $\geq 40 \%$ (valor predictivo positivo $86 \%$, IC $95 \% 78-94$ ). El análisis de los 12 pacientes clasificados incorrectamente demostró que el error se correlacionaba con la existencia de un infarto de miocardio de tipo no $Q$ en el $50 \%$ de ellos. La aplicación del algoritmo incorporando como quinta variable la presencia de infarto no $Q$ de localización anterior incrementó el valor predictivo positivo al $91 \%$.

\section{Conclusiones}

La aplicación de este algoritmo en pacientes post-infarto demostró un valor predictivo del $86 \%$ para identificar una fracción de eyección $\geq 40 \%$. La adición de una quinta variable (infarto no $Q$ de cara anterior) incrementó su valor predictivo.

Palabras Clave

Infarto de miocardio ; Fracción de Eyección ; Ecocardiografía ; Ventriculograma Radioisotópico, Electrocardiografía.

\section{COMENTARIO}

La estratificación del riesgo es esencial para el manejo de un paciente luego de un evento coronario agudo. La edad, la función ventricular (o su inversa: tamaño de la necrosis), la existencia de isquemia inducible residual y la existencia de arritmia ventricular son los indicadores pronósticos más importantes de la evolución luego de un infarto agudo de miocardio ${ }^{1-2}$. La evaluación de la función ventricular por medio de la fracción de eyección es por este motivo de empleo rutinario en el manejo post-infarto, habiéndose establecido en numerosos trabajos que un valor $~ 35-40 \%$ constituye un potente marcador de evolución adversa ${ }^{2}$. La medición de la misma requiere sin embargo de la utilización de métodos complementarios. En los años previos a la aparición de los mismos en la práctica médica se postuló el uso de diversos criterios electrocardiográficos con la finalidad de incrementar la sensibilidad para la detección de la magnitud del territorio necrótico ${ }^{3-4}$. La irrupción de la era de la "costo - efectividad" en la práctica médica motivó la utilización racional de los recursos. Este hecho fomentó la búsqueda de marcadores indirectos de sencilla implementación para estimar la función ventricular. El presente trabajo se orienta en ese sentido, pero:

1) La función ventricular residual pudo ser estimada indirectamente mediante el empleo del algoritmo propuesto en sólo 34,7\% de los pacientes, en un trabajo de diseño retrospectivo.

2) El error predictivo en la población "no predecible", así como en los pacientes con infarto no $Q$ fue superior al margen de seguridad necesario para un adecuado manejo y toma de decisión clínica.

En resumen, es interesante el retorno a los métodos indirectos de estimación de la función ventricular, pero la utilización del algoritmo propuesto no llega a "validarse" por el presente trabajo, que además nos muestra un inaceptable error predictivo. Por este motivo no es recomendable su aplicación clínica en su estado actual de desarrollo.

\section{Dr. Ernesto Ferreirós}

Servicio de Cardiología. Hospital Italiano de Buenos Aires.

Referencias

1- ACC/AHA Guidelines for the management of patients with acute myocardial infarction. A report of the ACC/AHA Task Force on Practice Guidelines (Committee on management of acute myocardial infarction). J. Am. Coll. Cardiol. 1996, 28: 1328-1428

2- McNamera RF, Carleen E, Moss AJ, y col. Estimating left ventricular ejection fraction after myocardial infarction by various clinical parameters. Am. J. Cardiol. 1988, 62: 192-196

3- Palmeri ST, Harrison DG, Cobb FR, y col. A QRS scoring system for assessing left ventricular function after myocardial infarction. N. Engl. J. Med. 1982, 306: 4-9

4- Selvester RH, Wagner GS, Hindeman NB, y col. The Selvester QRS scoring system for estimating myocardial infarct size. Arch. Intern. Med. 1985, 145: 1877-1881 